\title{
COUNTERROTATION IN GALAXIES
}

\author{
F. BERTOLA AND E.M. CORSINI \\ Dipartimento di Astronomia, Università di Padova \\ vicolo dell'Osservatorio 5, I-35122 Padova, Italy
}

\section{Introduction}

The phenomenon of counterrotation is observed when two galaxy components have their angular momenta projected antiparallel onto the sky. It follows that if the two components rotate around the same axis, the counterrotation is intrinsic. On the contrary the counterrotation is only apparent if the rotation axes are misaligned and the line-of-sight lies in between the two vectors or their antivectors. In the case of intrinsic counterrotation the two components can be superimposed or radially separated.

As far as the two components are concerned, stars are observed to counterrotate with respect to other stars or gas. The counterrotation of gas versus gas has been also detected. Up to now, the number of galaxies exhibiting these phenomena are $\sim 60$, with morphological types ranging from ellipticals to S0's and to spirals. Previous reviews about counterrotation are those of Rubin (1994b) and Galletta (1996).

When a second event occurs in a galaxy, such as the acquisition of material from outside, it is likely that the resulting angular momentum of the acquired material is decoupled from the angular momentum of the preexisting galaxy. Counterrotation is therefore a general signature of material acquired from outside the main confines of the galaxy. Good examples of such cases are ellipticals with a dust lane or gaseous disk along the minor axis and polar ring galaxies, where the angular momenta are perpendicular. It should be noted that recently attempts have been made to explain special cases of stars versus stars counterrotation in disk galaxies as due to a self-induced phenomenon in non-axisymmetric potentials (Evans \& Collett 1994, Wozniak \& Pfenninger 1997).

In the following we discuss the phenomenon according to the morphological type and to the kind of counterrotation. 


\section{Elliptical Galaxies}

\subsection{GAS VS. STARS}

Disks of ionized gas, which appear as dust lanes crossing the stellar body when seen on edge, have been detected in a large fraction $(\sim 50 \%)$ of ellipticals (e.g. Macchetto et al. 1996). Typical masses of dust and ionized gas in ellipticals are $M_{\text {dust }}=10^{5}-10^{6} M_{\odot}$ and $M_{\mathrm{HII}}=10^{3}-10^{5} M_{\odot}$ (e.g. Bregman et al. 1992). The kinematical decoupling generally observed (Bertola et al. 1990) between the gaseous and the stellar components suggests the gas is settled or in the process of settling in the equilibrium configurations. In triaxial ellipticals they are the planes orthogonal to the shortest and to the longest axes.

Intrinsic counterrotation of gas and stars in ellipticals has been observed in Anon 1029-459 (Bertola et al. 1988), IC 2006 (Schweizer et al. 1989), NGC 3528 (Bertola et al. 1988), NGC 5354 (Bettoni et al. 1995), NGC 5898 (Bettoni 1984, Bertola \& Bettoni 1988), and NGC 7097 (Caldwell et al. 1986).

\subsection{STARS VS. STARS}

Kinematically decoupled cores are observed in ellipticals when their stellar velocity field show a discontinuity between the rotation of the inner and the outer regions. Isolated cores have been detected in 17 ellipticals (Mehlert et al. 1997 and references therein) and in IC 4889 (Bertola et al. 1992). About half of them are stellar nuclear disks (Carollo et al. 1997).

Stellar counterrotation characterizes IC 1459 (Franx \& Illingworth 1988), IC 4889 (Bertola et al. 1992), NGC 1439, NGC 1700 (Franx et al. 1989), NGC 4472 (Davies \& Birkinshaw 1988), and NGC 3608 (Jedrzejewsky \& Schecther 1988), NGC 4816 (Mehlert et al. 1997), NGC 5322 (Bender 1988), NGC 7796 (Bertin et al. 1996). At least in the first five galaxies the counterrotation is intrinsic as indicated by the lack of velocity gradient along the minor axis. Minor axis observations are not available for the remaining four galaxies.

Several formation scenario have been proposed. They are the dissipationless minor merging with a compact low-luminosity elliptical (Kormendy 1984, Balcells \& Quinn 1990), the accretion of gas-rich dwarf companions and subsequent star formation as in dust-lane ellipticals (Franx \& Illingworth 1988), the dissipational major merging between an elliptical and a disk galaxy or between two disk galaxies (Schweizer 1990, Hernquist \& Barnes 1991), the hierarchical merging of dynamically hot, but still partially gaseous objects (Bender \& Surma 1992), and the interaction involving an elliptical with an embedded disk (Hau \& Thomson 1994). 


\subsection{GAS VS. GAS}

In the E4 galaxy NGC 1052 two dimensional high resolution $\mathrm{H} \alpha$ spectroscopy has revealed in the central $\pm 2 \mathrm{kpc}$ two apparently counterrotating ionized gas components (Plana \& Boulesteix 1996). The two components are interpreted as produced by two distinct structures superimposed by a projection effect and settled onto the two orthogonal equilibrium planes of a triaxial elliptical galaxy. They have a mass of $M_{\mathrm{HII}, 1} \sim 10^{3} M_{\odot}$ and $M_{\mathrm{HII}, 2} \sim 10^{5} M_{\odot}$ respectively.

The case of the E5 galaxy IC 4889 (Vega et al. 1997, these proceedings) is more complex. In addition to the above mentioned counterrotating core, two gas disks settled onto the allowed planes of a triaxial galaxy apparently counterrotate. Both cases indicate multiple acquisition events.

\section{Disk Galaxies}

\subsection{GAS VS. STARS}

A compilation of $36 \mathrm{~S} 0$ galaxies with ionized gas based on the samples of Bertola et al. (1992) and of Kuijken et al. (1996) lists 12 objects with gas counterrotating with respect to the stars. The large fraction of S0's with kinematical decoupling between stars and gas is consistent with the idea that the gas in lenticulars is acquired from outside (Bertola et al. 1992). Episodic infall of external gas, continuous infall of external gas and dissipational merging with a gas-rich dwarf companion all have been investigated by means of numerical simulation (Thakar \& Ryden 1996, Thakar et al. 1997) as viable mechanisms for producing an overall gaseous counterrotation in disk galaxies. In the infall case the acquisition rate, the initial angular momentum and the gas clumpiness state are crucial parameters to successfully build up the counterrotating gas disk without dynamically heating the preexisting stellar disk. The same is true for the mass of the captured satellite in the merging case.

In the edge-on peanut S0 NGC 128 (Emsellem \& Arsenault 1997) a tilted gaseous disk counterrotates with respect to the stars. It has been interpreted as due to acquired gas settled onto the so-called anomalous orbits in a tumbling triaxial potential, as in the case of the barred S0 NGC 2217 (Bettoni et al. 1990) and NGC 4684 (Bettoni et al. 1993).

In the early Sa NGC 3626 (Ciri et al. 1995) the neutral, ionized and molecular gas (Garcia-Burillo et al. 1997), amounting to $\sim 10^{9} M_{\odot}$, counterrotates at all radii with respect to the stars. It should be noted that, in spite of the presence of two faint amorphous dust and possibly stellar spiral arms, the morphology of NGC 3626 resembles that of more typical S0 galaxies. 


\subsection{STARS VS. STARS}

\subsubsection{Stellar Disk vs. Stellar Disk}

The S0 galaxy NGC 4550 has two cospatial counterrotating stellar disks, one of them corotating with the gaseous one (Rubin et al. 1992). The two disks have exponential surface brightness profiles with the same central surface brightnesses and scale lengths (Rix et al. 1992). In the early-type spirals NGC 4138 (Jore et al. 1996) and NGC 7217 (Merrifield \& Kuijken 1994) about $20 \%-30 \%$ of the stars in the disk are in retrograde orbits constituting a kinematically distinct component. Both disks are equally extended but have different levels of surface brightness. In NGC 7217 the gaseous disk rotates in the same direction as the primary (i.e. the more massive) stellar disk. The contrary is true for NGC 4138.

The presence of a counterrotating stellar disk is interpreted as the result of a subsequent stellar formation in an accreted gaseous disk. In the case of NGC 7217 where the ionized gas rotates in the same direction as the more massive disk, Merrifield and Kuijken (1994) suggested that the actual retrograde (i.e. the less massive) disk formed first. An alternative to the second event scenario has been proposed for NGC 4550 by Evans \& Collett (1994). They stated that whenever oval disks become circular or triaxial halos in which they are embedded become axisymmetric, then box orbits are scattered equally into clockwise and counterclockwise rotating tube orbits. In this way two identical counterrotating stellar disks can be built. The presence of three gaseous rings in NGC 7217 has suggested to Athanassoula (1996), that in this galaxy the bar has decayed, causing the counterrotation of the less massive stellar disk.

Extended stellar counterrotation in disks of S0s and spirals seems to be a rare phenomenon according to the results of Kuijken et al. (1996). They measured carefully the line-of-sight velocity distribution along the major axes of $28 \mathrm{~S} 0$ galaxies, without finding any new case. Indeed they estimated that less than $10 \%$ of S0's show large-scale counterrotation with more than $5 \%$ of disk stars on retrograde orbits. If counterrotating stars formed from acquired gas, this result contrasts with the large fraction of S0's exhibiting counterrotating disks of ionized gas. However, most of these gas disks are too small to produce the large-scale counterrotation observed in systems like NGC 4138, NGC 4550 and NGC 7217.

The Sa galaxy NGC 3593 (Bertola et al. 1996) is composed of a small bulge, a first, radially more concentrated, stellar disk that contains about $20 \%$ of the total luminous mass and dominates the star kinematics in the inner $\pm 1 \mathrm{kpc}$, and a second counterrotating stellar disk, radially more extended, dominating the outer kinematics. The two disks have exponential luminosity profiles with different scale lengths and central surface bright- 


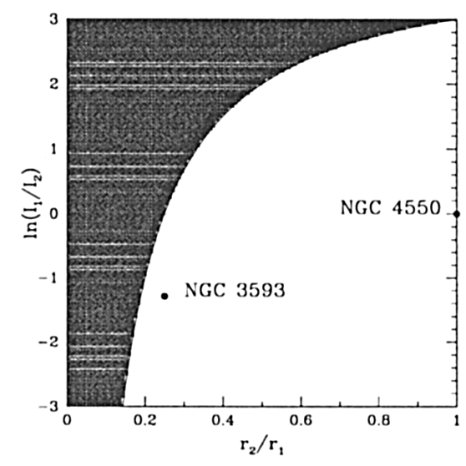

Figure 1. Detectability of two counterrotating stellar disks having exponential luminosity profiles of scale lengths $r_{1}$ and $r_{2}$ and central intensities $I_{1}$ and $I_{2}$. We assume to disentangle the two disks if the intensity of the smaller one (disk 2) is at least the $5 \%$ of that of the greater one (disk 1) at all radii lower than $r_{1}$ (non-hatched area). The dots correspond to the observed cases of NGC 4550 (Rubin et al. 1992, Rix et al. 1992) and NGC 3593 (Bertola et al. 1996).

nesses. The gaseous disk counterrotates with respect to the more extended disk. The existence of cases like NGC 3593, where two disks of different scale lengths and central surface brightnesses counterrotate, suggest that they can be numerous if not a general case. Fig. 1 shows the vast area to be explored in the plane central surface brightness vs. scale length within the limit of present day detectability.

Instabilities in disk galaxies with counterrotating stars and/or gas have been recently investigated by Sellwood \& Merritt (1994) and Lovelace et al. (1997). Due to the results to their numerical simulations Comins et al. (1997) suggest that one-armed spiral features may characterize disk galaxies with counterrotation. Spirals with tightly-coiled narrow arms are the candidates for Howard et al. (1997).

\subsubsection{Stellar Bulge vs. Stellar Disk}

In the Sb NGC 7331 (Prada et al. 1996) the bulge counterrotates with respect to the disk. The galaxy appears morphologically smooth and undisturbed, but the analysis of the stellar LOSVD reveals the presence in the inner $\pm 1.4 \mathrm{kpc}$ of two counterrotating stellar components. Near-infrared photometry shows that the radial surface brightness profile of the slowrotating component follows that of the bulge, while the fast-rotating follows the disk. Also the Sb spiral NGC 2841 seems to have a counterrotating bulge (Prada 1997, private communication).

Did disk galaxies really start out as 'undressed spheroids' and the disks formed gradually over several several billion of years as suggest by Binney 
\& May (1986)? To answer this question we need to know how unique are the cases of NGC 2841 and NGC 7331 . The absence of a velocity gradient in the central $( \pm 1 \mathrm{kpc})$ regions of the Sa NGC 4698 would suggest the presence of a counter or orthogonally rotating bulge (Bertola et al. 1997, in preparation).

\subsubsection{Stellar Counterrotation in Early-Type Barred Galaxies}

The stellar rotation curve along the major axis of the bar of eleven earlytype barred galaxies seen at intermediate inclination show a 'waving pattern'. Nine are listed by Bettoni \& Galletta (1997) while two, namely NGC 5005 and NGC 5728 were found by Prada (1997, private communication). The observed amplitude of the oscillations is lower than $30 \mathrm{~km} \mathrm{~s}^{-1}$ producing in the rotation curve a region of minimum, which sometimes can reach negative values.

The phenomenon, which was pointed out by Bettoni (1989), has been recently interpreted by Wozniak \& Pfenninger (1997). They used self-consistent models of barred galaxies with reproduce the observed wavy pattern as due to the presence of retrograde orbits with a local concentration in the region of minimum velocity. The origin of such a stellar counterrotation is not necessarily external, as acquired gas can be trapped on this family of retrograde orbits and then eventually form stars. These counterrotating gas and stars could produce an embedded retrograde secondary bar. Friedli (1996) demonstrated that galaxies having two nested and counterrotating bars are stable and long-lived systems. He also predicted the peculiar signatures characterizing their kinematics, which is not yet observed.

\subsection{GAS VS. GAS}

NGC 4826, a nearby and relatively isolated Sab(s) spiral called 'Devil-Eye' or 'Black-Eye' contains two nested counterrotating gaseous disks (Braun et al. 1992). Radio and optical observations (Braun et al. 1994, Rubin 1994a) revealed an inner disk of about $1 \mathrm{kpc}$ radius containing $\sim 10^{7} M_{\odot}$ in $\mathrm{H} \mathrm{I}$ and $\sim 10^{8} M_{\odot}$ in $\mathrm{H}_{2}$ and a counterrotating outer gas disk extending from 1.5 to $11 \mathrm{kpc}$ and containing $\sim 10^{8} M_{\odot}$ in $\mathrm{H}$ I. They are coplanar to the stellar disk. Stars corotate with the inner gas, but beyond the dust lane less than $5 \%$ of them $\left(\sim 10^{8} M_{\odot}\right)$ corotate with the outer gas (Rix et al. 1995). The kinematical features of NGC 4826 are interpreted (Rix et al. 1995) considering an original gas-poor galaxy with prograde gas which slowly acquires a comparable mass of external retrograde gas. The new counterrotating gas settles in the outer parts of the stellar disk, leaving undisturbed the galaxy morphology (Walterbos et al. 1994). Another case we interpret as constituted by two counterrotating gas components as in 
NGC 4826, is the edge-on S0 NGC 5252 (Held et al. 1992)

The late-type galaxy NGC 253 (Anantharamaiah \& Goss 1996) contains in the central $\sim 150 \mathrm{pc}$ region three nested structures of ionized gas: a counterrotating inner disk, an orthogonal rotating ring and an outer ring rotating in the same sense as the galactic disk.

In the edge-on S0 NGC 7332 Fisher et al. (1994) detected a second gas component corotating with the stars in addition to the main counterrotating gaseous disk. Recently two dimensional high resolution $\mathrm{H} \alpha$ spectroscopy (Plana \& Boulesteix 1996) has revealed that the two ionized gas counterrotating structures are apparently superimposed. They extend to about 4 kpc and contain $\sim 10^{5} M_{\odot}$ in $\mathrm{H}$ II. The accretion of newly supplied counterrotating gas onto an existing corotating gas disk investigated by Lovelace \& Chou (1996) could be a possible formation scenario for NGC 7332.

\section{Conclusions}

Counterrotation occurs in a wide variety of forms (gas vs. stars, stars vs. stars, gas vs. gas). It is present in galaxies of different morphological types, ranging from ellipticals to early-type disk galaxies.

The counterrotation of stellar vs. stellar disks is the type of counterrotation we expect to be prevailing, since it is the end result of gas vs. stars counterrotation. Therefore its frequency could be very high if the area described in Fig. 1 is carefully inspected, using the state of the art analysis of the shape and asymmetries of the line profiles of the absorption lines. This would indicate that acquisition and merging events are common phenomena in the history of galaxies.

We are grateful to D. Burstein for useful comments on the manuscript.

\section{References}

Anantharamaiah, K.R., Goss, W.M. 1996, ApJ, 466, L13

Athanassoula, E. 1996, In: R. Buta, D.A. Crocker, B.G. Elmegreen (eds.) ASP Conf. Ser. 91, "Barred Galaxies". ASP, San Francisco, p. 309

Balcells, M., Quinn, P.J. 1990, ApJ, 361, 381

Bender, R., 1988, A\&A, 202, L5

Bender, R., Surma, P. 1992, A\&A, 258, 250

Bertin, G., Bertola, F., Buson, L.M., Danziger, I.J., Dejonghe, H., Sadler, E.M., Saglia, R.P., de Zeeuw, P.T., Zeilinger, W.W. 1994, A\&A, 292, 381

Bertola, F., Bettoni, D. 1988, ApJ, 329, 102

Bertola, F., Bettoni, D., Buson, L.M., Zeilinger, W.W. 1990, In: R. Wielen (ed.) "Dynamics and Interactions of Galaxies". Springer-Verlag, Berlin, p. 249

Bertola, F., Buson, L.M., Zeilinger, W.W. 1988, Nature, 335, 705

Bertola, F., Buson, L.M., Zeilinger, W.W. 1992, ApJ, 401, L79

Bertola, F., Cinzano, P., Corsini, E.M., Pizzella, A., Persic, M., Salucci, P. 1996, ApJ, 458, L67

Bettoni, D. 1984, The Messenger, 37, 17 
Bettoni, D. 1989, AJ, 97, 79

Bettoni, D., Galletta, G. 1997, A\&AS, 124, 61

Bettoni, D., Buson, L.M., Maira, L., Bertola, F. 1995, In: Richter O.G., Borne K. (eds.) ASP Conf. Ser. 70, "Groups of Galaxies". ASP, San Francisco, p. 95

Bettoni, D., Fasano, G., Galletta, G. 1990, AJ, 99, 1789

Bettoni, D., Galletta, G., Sage, L.J. 1993, A\&A, 280, 121

Binney, J.J., May, A. 1986, MNRAS, 218, 743

Braun, R., Walterbos, R.A.M., Kennicutt Jr., R.C. 1992, Nature, 360, 442

Braun, R., Walterbos, R.A.M., Kennicutt Jr., R.C., Tacconi, L.J. 1994, ApJ, 420, 558

Bregman, J.N., Hogg, D.E., Roberts, M.S. 1992, ApJ, 387, 484

Caldwell, N., Kirshner, R.P., Richstone, D.O. 1986, ApJ, 305, 136

Carollo, C.M., Franx, M., Illingworth, G.D., Forbes, D.A. 1997, ApJ, 481, 710

Ciri, R., Bettoni, D., Galletta, G. 1995, Nature, 375, 661

Comins, N., Lovelace, R.V.E., Zeltwanger, T., Shorey, P. 1997, ApJ, 484, L33

Davies, R.L., Birkinshaw, M. 1988, ApJS, 68, 409

Emsellem, E., Arsenault, R. 1997, A\&A, 318, L39

Evans, N.W., Collett, J.L. 1994, ApJ, 420, L67

Fisher, D., Illinghworth, G.D., Franx, M. 1994, AJ, 107160

Franx, M., Illinghworth, G.D. 1988, ApJ, 327, L55

Franx, M., Illinghworth, G.D., Heckmann, T. 1989, ApJ, 344, 613

Friedli, D. 1996, A\&A, 312, 761

Galletta, G. 1996, In: R. Buta, D.A. Crocker, B.G. Elmegreen (eds.) ASP Conf. Ser. 91, "Barred Galaxies". ASP, San Francisco, p. 429

Garcia-Burillo, S., Sempere, M.J., Bettoni, D. 1997, ApJ, submitted

Hau, G.K.T., Thomson, R.C. 1994, MNRAS, 270, L23

Held, E.V., Capaccioli, M., Cappellaro, E. 1992, In: S.S. Holt, S.G. Neff, C.M. Urry (eds.)

AIP Conf Proc. 254 "Testing the AGN Paradigm". AIP, New York, p. 613

Hernquist, L., Barnes, J.E. 1991, Nature, 354, 210

Howard, S., Carini, M.T., Byrd, G.G., Lester, S. 1997, AJ, submitted

Jedrzejewsky, R., Schecther, P.L. 1988, ApJ, 330, L87

Jore, K.P., Broelis, A.H., Haynes, M. 1996, AJ, 112, 438

Kormendy, J. 1984, ApJ, 287, 577

Kuijken, K., Fisher, D., Merrifield, M.R. 1996, MNRAS, 283, 543

Lovelace. R.V.E., Chou, T. 1996, ApJ, 468, L25

Lovelace, R.V.E., Jore, K.P., Haynes, M.P. 1997, ApJ, 475, 83

Macchetto, F., Pastoriza, M., Caon, N., Sparks, W.B., Giavalisco, M., Bender, R., Capaccioli, M. 1996, A\&AS, 120, 463

Mehlert, D., Saglia, R.P., Bender, R., Wegner, G. 1997, A\&A, submitted

Merrifield, M.R., Kuijken, K. 1994, ApJ, 432, 575

Plana, H., Boulesteix, J. 1996, A\&A, 307, 391

Prada, F., Gutierrez, C.M., Peletier, R.F., McKeith, C.D. 1996, ApJ, 463, L9

Rix, H.-W., Franx, M., Fisher, D., Illingworth, G. 1992, ApJ, 400, L5

Rix, H.-W., Kennicutt Jr., R.C., Braun, R., Walterbos, R.A.M. 1995, ApJ, 438, 155

Rubin, V.C. $1994 \mathrm{a}$, AJ, 107, 173

Rubin, V.C. $1994 \mathrm{~b}, \mathrm{AJ}, 108,456$

Rubin, V.C., Graham, J.A., Kenney, J.D.P. 1992, ApJ, 394, L9

Schweizer, F. 1990, In: R. Wielen (ed.) "Dynamics and Interactions of Galaxies". Springer-Verlag, Berlin, p. 60

Schweizer, F., van Gorkom, J.H., Seitzer, P. 1989, ApJ, 338, 770

Sellwood, J.A., Merritt, D. 1994, ApJ, 425, 530

Thakar, A., Ryden, B. 1996, ApJ, 461, 55

Thakar, A., Ryden, B., Jore, K.P., Broeils, A.H. 1997, ApJ, 479, 702

Walterbos, R.A.M., Braun, R., Kennicutt Jr., R.C. 1994, AJ, 107, 184

Wozniak, H., Pfenninger, D. 1997, A\&A, 317, 14 